07,10

\title{
О некоторых принципиальных особенностях обработки данных испытаний на откольное разрушение
}

\author{
(C) Г.А. Волков ${ }^{1,2}$, Ю.В. Петров ${ }^{1,2}$, А.А. Уткин ${ }^{1,2}$ \\ ${ }^{1}$ Санкт-Петербургский государственный университет, \\ Санкт-Петербург, Россия \\ ${ }^{2}$ Институт проблем машиноведения РАН, \\ Санкт-Петербург, Россия \\ E-mail: volkovgrig@mail.ru, yp@yp1004.spb.edu
}

(Поступила в Редакцию 20 июня 2016 г.)

\begin{abstract}
Анализируется способ обработки результатов динамических испытаний по откольному разрушению, основанный на точном решении волнового уравнения, а также получивший широкое распространение его упрощенный вариант, основанный на предполагаемой однозначной связи величины сброса скорости свободной поверхности с предельным напряжением разрыва среды. При помощи рассматриваемых точных решений волновой методики определяются возникающие при отколе импульсы растягивающих напряжений. Приводится сравнение полученных уровней напряжений в момент разрушения со значениями откольной прочности, вычисленными по методике сброса скорости. Показаны случаи совпадения и расхождения результатов, полученных с помощью обеих методик. На примере нагружающих импульсов различной формы приведены возможные сценарии разрушения образца, в частности, показана возможность возникновения эффекта задержки разрушения, который может быть потерян при упрощенном способе обработки.
\end{abstract}

Работа выполнена при поддержке грантов РФФИ 14-01-00814, 16-51-53077, 16-01-00638 гранта СПбГУ 6.38.243.2014, а также гранта Президента Российской Федерации МК-7596.2015.1.

DOI: 10.21883/FTT.2017.02.44052.248

В настоящее время одним из основных способов динамического испытания материалов на растяжение в микро- и субмикро-секундном диапазоне длительностей является эксперимент, основанный на явлении откольного разрушения. При соответствующих испытаниях в образце инициируется сжимающий импульс напряжений, который при отражении от свободной поверхности приводит к единичному или множественному разрыву материала [1-3]. Информацию о динамических процессах, происходящих в образце во время испытания, получают измерением скорости частиц свободной поверхности. Было разработано несколько различных способов измерения, которые позволяют получить временну́ю зависимость скорости свободной поверхности с разрешением вплоть до десятков пикосекунд [4,5]. Это позволяет детально зафиксировать все высокоскоростные изменения, возникающие перед разрушением образца. Обычно при интерпретации полученных данных на временных профилях скорости свободной поверхности удается выделить несколько характерных точек, указывающих на возникновение в материале упругой волны, пластической волны, фазовых превращений, а также момента разрушения образца и координату откольного сечения [6-11]. В частности, по этим точкам определяют величину откольной прочности материала, под которой обычно подразумевается величина растягивающих напряжений в месте разрыва на момент разрушения.

В настоящее время часто применяется способ определения динамической прочности по так называемому сбросу скорости - разности между максимальным значением и последующим за ним первым локальным минимумом на диаграмме скорости свободной поверхности [7-11]. Данный способ может оказываться вполне эффективным в условиях, когда в образцах инициируются высокоинтенсивные запороговые воздействия [11]. Однако об этой особенности не следует забывать при обработке экспериментальных данных в иных условиях. Так, в случае пороговых разрушающих импульсов вычисление откольной прочности по сбросу скорости может привести к некорректным результатам. Это связано с тем, что динамическая прочность материала не может быть однозначно охарактеризована некоторым критическим уровнем напряжений, пусть даже и отличающимся для различных скоростей нагружения. При исследовании динамической прочности важно учитывать не только максимальный уровень напряжений, но также форму и длительность воздействия [12-14].

Классическая методика обработки, основанная на решении волновой задачи, подразумевает восстанавливать полностью временной профиль разрушающих напряжений [1-3]. Суть методики наиболее наглядно можно продемонстрировать на примере простейшего одномерного случая для линейно-упругой среды. На отрезке $0<x<L$, моделирующем упругую полосу, требуется найти решение волнового уравнения для перемещения частиц среды $w=w(t, x)$

$$
\frac{1}{c^{2}} \frac{\partial^{2} w}{\partial t^{2}}-\frac{\partial^{2} w}{\partial x^{2}}=0
$$


при граничных условиях на концах интервала для функции нормального напряжения

$$
\left.\sigma\right|_{x=0}=0,\left.\quad \sigma\right|_{x=L}=-P g(t),
$$

и нулевых начальных условиях

$$
\left.w\right|_{t=0}=\left.\frac{\partial w}{\partial t}\right|_{t=0}=0,
$$

где $P$ - амплитуда ударного импульса „давления“ на правой поверхности, $\sigma=\rho c^{2} \frac{\partial w}{\partial x}-$ нормальное напряжение; $c, \rho-$ скорость волны и плотность среды соответственно. Функция формы импульса $0 \leq g(t) \leq 1$ считается отличной от нуля на некотором временном интервале $0<t<T$, задающем длительность удара.

Для удобства дальнейшего анализа и сопоставления с традиционно проводимыми измерениями предполагается, что время отсчитывается от момента начала отражения волнового импульса от свободной поверхности $x=0$. Тогда точное решение задачи для нормального напряжения на временном интервале $-L / c<t<L / c$ определяется суммой отраженной и прямой волн $\sigma=P\left(g\left(t-\frac{x}{c}\right)-g\left(t+\frac{x}{c}\right)\right)$, или в терминах, измеряемых на свободной поверхности величин, выражением

$$
\sigma=\frac{1}{2} \rho c\left(f\left(t-\frac{x}{c}\right)-f\left(t+\frac{x}{c}\right)\right),
$$

где функция $f(t)=\frac{2 P}{\rho c} g(t)$ описывает временной профиль скорости частиц среды, фиксируемый (например, при помощи техники интерферометрии $[4,5])$ на свободной поверхности. Далее данная методика, в силу своих особенностей, будет условно называться волновой.

В настоящее время получил широкое распространение способ оценки уровня напряжений в момент разрушения по величине „сброса скорости“ на свободной поверхности мишени. В этом случае величина откольной прочности определяются следующим образом

$$
\sigma_{s}=\frac{1}{2} \rho c \Delta f
$$

где $\Delta f=f_{1}-f_{2}-$ разность между максимальным значением функции скорости свободной поверхности и ее значением в первой точке локального минимума, которая соответствует моменту прихода сигнала об образовании откольной трещины на свободную поверхность (рис. 1). Иногда в качестве вычитаемого берется средняя скорость частиц свободной поверхности в откольной волне.

Цель дальнейшего анализа - показать, что найденная по методу „сброса скорости“ (5) прочность $\sigma_{s}$ не всегда будет совпадать с напряжением в сечении откола в момент разрушения $\sigma_{r}$, полученным по точному решению вышеприведенной волновой задачи. Особенно важным и существенным будет отличие в случае пороговых воздействий, когда, как показано в ряде экспериментов, разрушение может происходить с задержкой, т.е. после достижения максимальных значений растягивающего напряжения в откольном сечении. Экспериментально явление задержки разрушения может наблюдаться не только при отколе [15], но также и в случае разрушения образцов с трещинами [16].

Для простоты производимых вычислений разница результатов, получаемых при расчете откольной прочности по сбросу скорости или с помощью волновой методики, будет проиллюстрирована на примере импульсов напряжений треугольной и трапецеидальной формы. Такой выбор временного профиля также объясняется тем, что он приближенно соответствует типовым импульсам, наблюдаемым экспериментально. Пусть для треугольных импульсов продолжительности роста и спада нагрузки будут обозначаться через $T_{1}$ и $T_{2}$ соответственно. Таким образом, общая продолжительность воздействия $T=T_{1}+T_{2}$. Предполагается, что разрушение наступает в момент времени $t_{r}$, при этом координата откольного сечения $x_{r}$. Также подразумевается, что откольное сечение возникает мгновенно (в момент времени $t_{r}$ ) и образует новую свободную от напряжений поверхность.

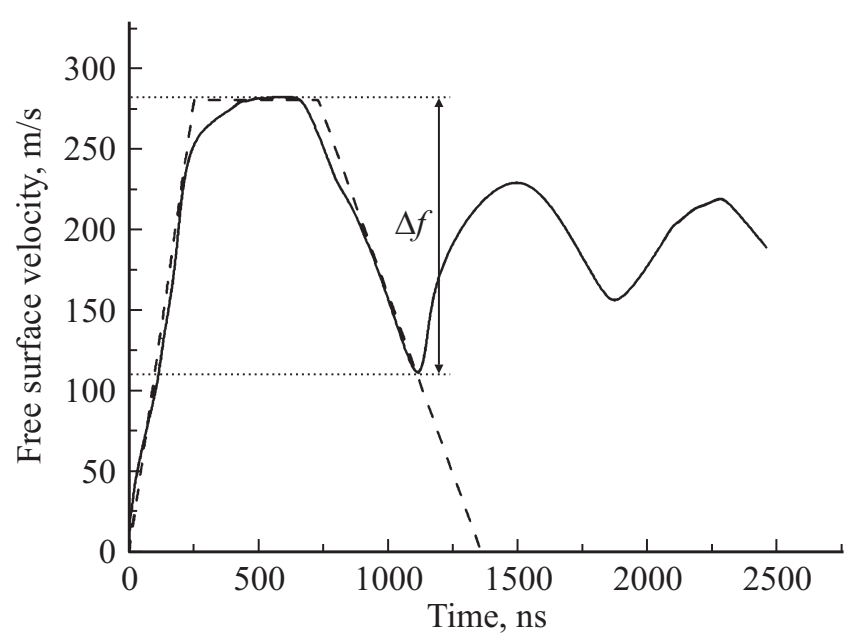

Рис. 1. Типовой профиль скорости частиц свободной поверхности.

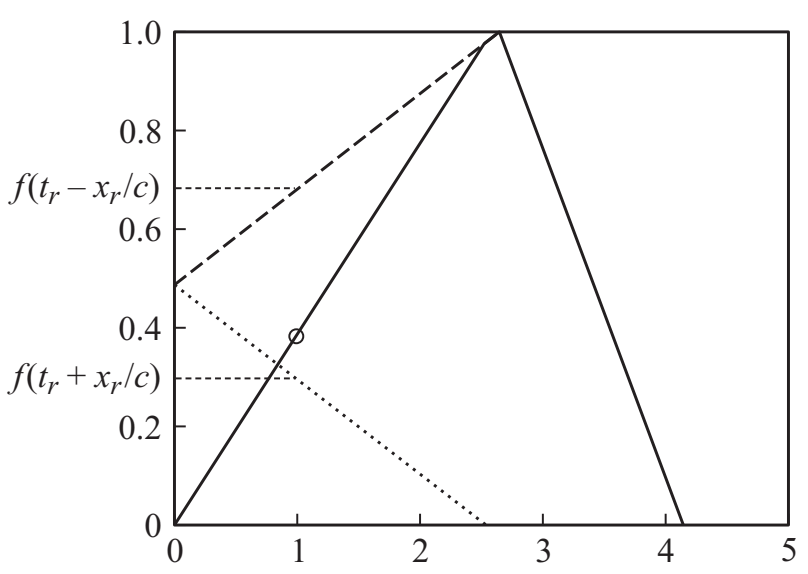

Рис. 2. Зависимость скорости частиц в волне от координаты в момент разрушения. 
$a$

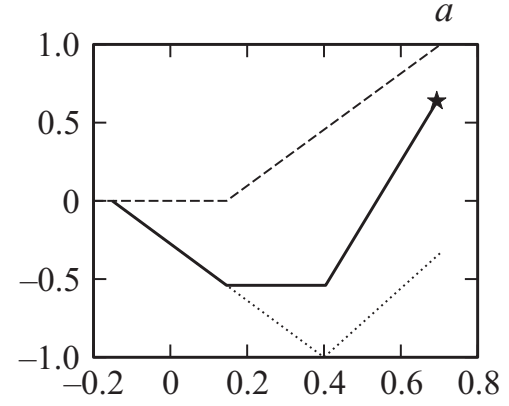

$b$

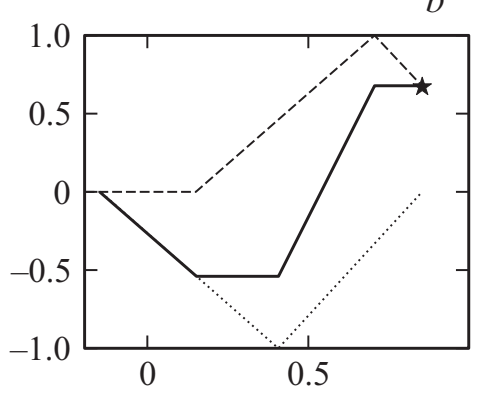

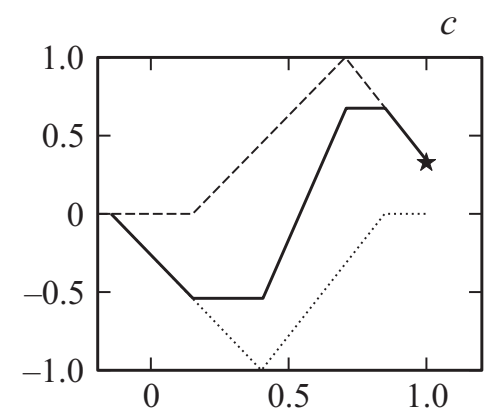

Рис. 3. Временна́я зависимость напряжений в откольном сечении. Звездочкой отмечен момент разрушения.

На рис. 2. показана зависимость скорости в волне от координаты в некоторый возможный момент разрушения. Скорость и координата нормированы, точками обозначена падающая волна, пунктиром - отраженная, сплошная линия обозначает суммарное действие волн. В соответствии с соотношением (4) в откольном сечении напряжение будет определяться разностью профилей отраженной $f\left(t-x_{r} / c\right)$ и падающей $f\left(t+x_{r} / c\right)$ волн. Часть волнового профиля с координатами $t_{r}<t<t_{r}+x_{r} / c$ движется к свободной поверхности в виде сжимающей волны, а другая часть с координатами $t_{r}-x_{r} / c<t<t_{r}$ движется в обратном направлении от свободной поверхности, создавая в образце поле растягивающих напряжений. После разрушения растягивающая волна, отразившись от откольного сечения, в момент времени $t=t_{r}+x_{r} / c$ возвращается к свободной поверхности, тем самым сообщая о произошедшем ранее разрушении.

Расчет по точному решению показывает, что при варьировании координаты гипотетического откольного сечения $x_{r}$ будет изменяться разность между величинами $t_{r}-x_{r} / c$ и $t_{r}+x_{r} / c$. Таким образом, чем дальше от свободной поверхности находится рассматриваемое сечение, в котором возможно возникновение откола, тем более высокий уровень растягивающих напряжений может в нем достигаться при прочих равных условиях. При этом возможное время разрушения $t_{r}$ может принимать произвольные значения, при условии, что в этот момент откольное сечение находится под действием растягивающих напряжений. Таким образом, должно выполняться соотношение $f\left(t_{r}-x_{c} / c\right)>f\left(t_{r}+x_{c} / c\right)$. Для рассматриваемого случая с треугольным импульсом получается $t_{r}>T_{1}-\left(2 x_{r} / c\right)\left(T_{1} / T\right)$. Зависимость напряжений в откольном сечении от времени вплоть до момента разрушения в возможных ситуациях показана на рис. 3 . Точками обозначены напряжения в падающей волне, пунктиром - в отраженной, а сплошной линией суммарные. Напряжения и время нормированы относительно амплитуды и длительности воздействия. Элементарные геометрические преобразования показывают, что если $t_{r}-x_{r} / c<T_{1}$, то суммарное напряжение в сечении откола будет к моменту разрушения расти (рис. $3, a$ ); если $t_{r}+x_{r} / c<T$, то этому моменту будет предшество- вать участок с постоянным напряжением (рис. $3, b)$ и в противном случае напряжение к моменту разрушения будет уменьшаться (рис. $3, c)$.

Анализ истории напряжений в различных сечениях показывает, что между двумя способами по определению откольной прочности имеется заметное различие. Разница между уровнем напряжений, определенным волновым способом, и величиной откольной прочности, вычисленной по сбросу скорости, может достигать сотен процентов. При этом важно, что в данном случае под откольной прочностью имеется в виду лишь вычисленное по волновому полю значение напряжения в момент разрыва материала, но это событие не связывается с выполнением какого-либо критерия.

На рис. 4-6 показаны случаи с треугольными и трапецеидальными нагружающими импульсами, соответствующие возможному разрушению в сечениях, отстоящих на некоторое расстояние от свободной тыльной поверхности. Эти примеры выбраны для иллюстрации возможных сценариев, при которых результаты обеих методик могут, как полностью совпадать, так и существенно отличаться друг от друга. На рис. 4 сечение, в котором произошло разрушение, было выбрано таким образом, что результаты вычислений по сбросу и по классической методике полностью совпали.

В то же время для тех же нагружающих импульсов в других возможных откольных сечениях результаты расчетов существенно отличаются (рис. 5,6). Стоит отметить, что в проведенном анализе прочностные свойства испытываемого материала никак не оговаривались, поэтому гипотетическое разрушение возможно в любом сечении образца. Это означает, что при обработке реальных экспериментально полученных временных профилей скорости свободной поверхности может реализоваться любой из предложенных сценариев.

Для ответа на вопрос, в каких случаях результаты обеих методик совпадают, формулу (5) для вычисления откольной прочности по сбросу скорости можно переписать в следующем виде

$$
\sigma_{s}=\frac{1}{2} \rho c\left(\max _{t} f(t)-f\left(t_{r}+\frac{x_{r}}{c}\right)\right) .
$$



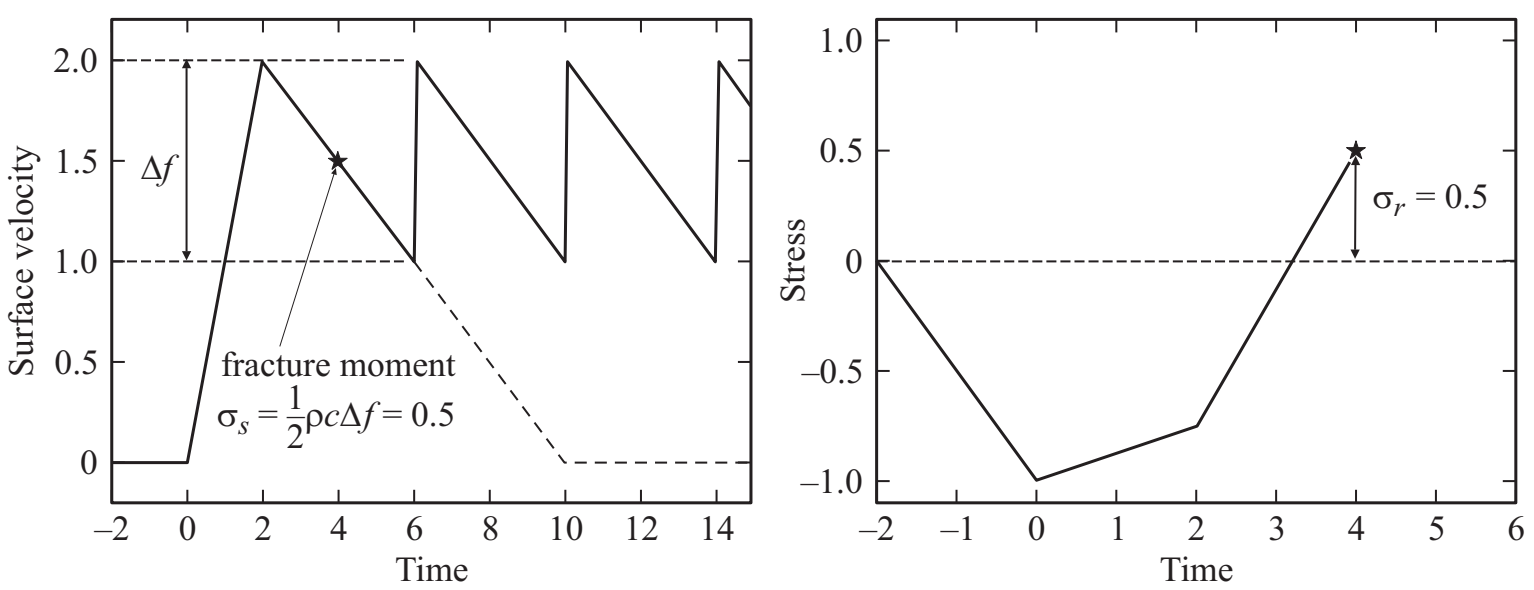

Рис. 4. Треугольный импульс. Откольная прочность, вычисленная по сбросу, совпадает с напряжениями, определенными на момент разрушения по классической методике.
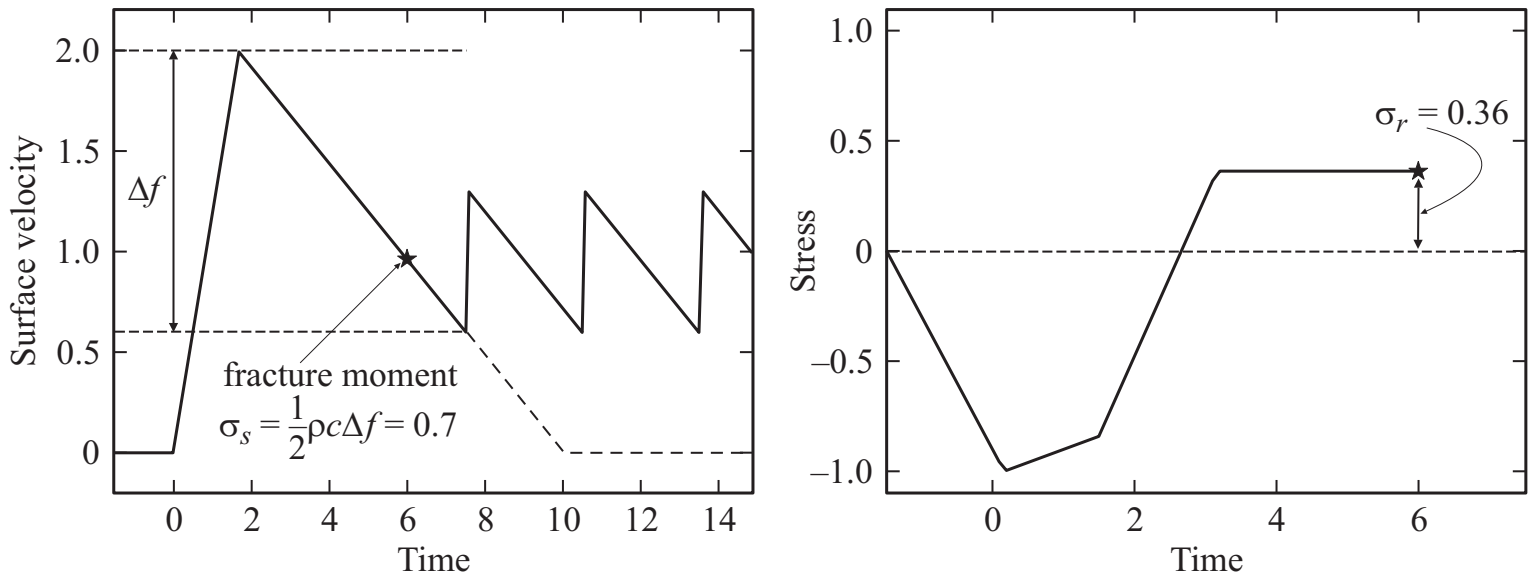

Рис. 5. Треугольный импульс. Откольная прочность, вычисленная по сбросу, превышает уровень напряжений, определенный на момент разрушения по классической методике.
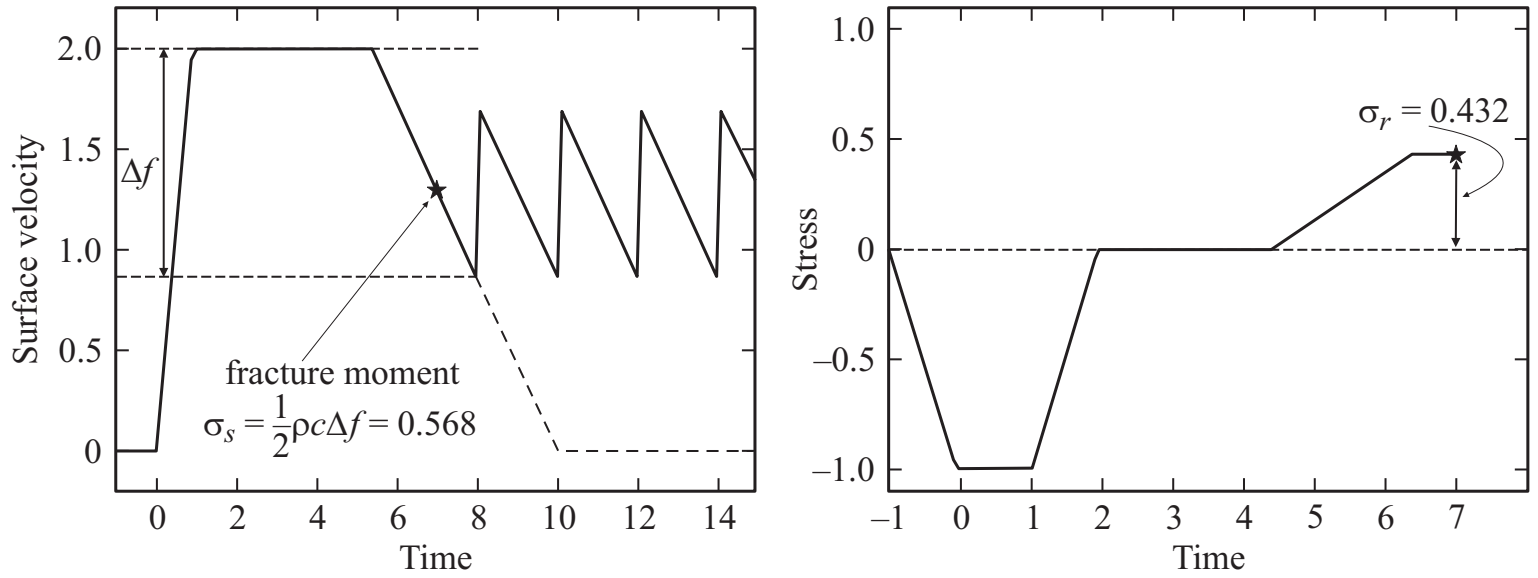

Рис. 6. Трапецеидальный импульс. Откольная прочность, вычисленная по сбросу, превышает уровень напряжений, определенный на момент разрушения по классической методике. 

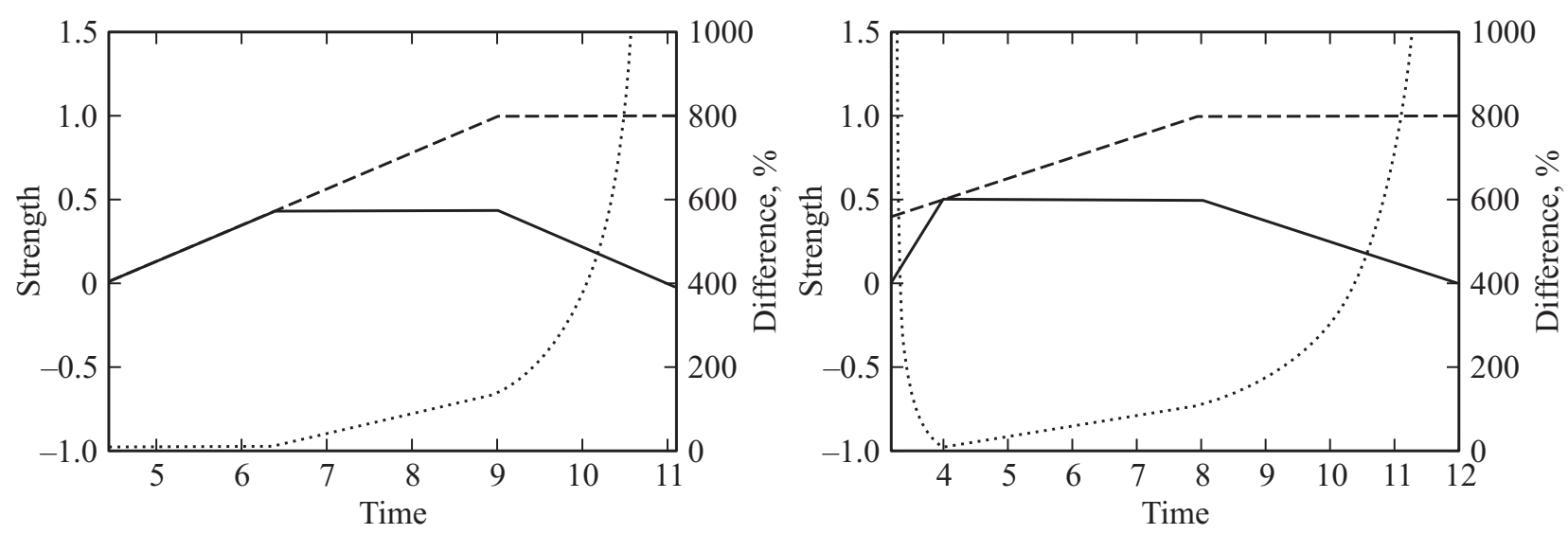

Рис. 7. Сравнение откольной прочности, вычисленной с помощью различных методик: сплошная линия - результат решения волновой задачи в упругой постановке, пунктирная - по сбросу скорости, линия из точек - относительная разница. $a-$ трапецеидальный импульс, $b$ - треугольный импульс.

Сравнение выражений (4) и (6) показывает, что величины $\sigma_{r}$ и $\sigma_{s}$ будут совпадать только в том случае, если значения $f\left(t_{r}-x_{r} / c\right)$ и $\max _{t} f(t)$ совпадают, т.е. если в момент времени $t_{r}-x_{r} / c$ скорость на свободной поверхности максимальна. Во всех остальных случаях прочность, определенная по сбросу будет превышать фактическую. Соответствующие графики приведены на рис. 7 для импульсов треугольной и трапецеидальной формы.

Здесь напряжения, полученные в результате решения волновой задачи в упругой постановке - сплошная линия, а прочность, определенная по сбросу - пунктирная линия. На правой оси ординат отложена относительная разница между полученными результатами.

Максимально возможные растягивающие напряжения, равные амплитуде приложенного импульса треугольной формы, впервые могут быть достигнуты в момент времени $t_{\max }=T_{1}+T_{2} / 2$ в сечении с координатой $x_{\max }=c T_{2} / 2$, при условии, что ранее при меньших напряжениях не было разрушения образца. Если предположить, что образец разрушается сразу же, при достижении максимального уровня напряжений, то в этом случае напряжения в сечении откола совпадают с определенными по сбросу. Однако перемещение свободной поверхности имеет нехарактерный вид. Скорость перемещения к моменту прихода сигнала о разрушении падает до нуля, чего обычно не наблюдается в экспериментах.

Рассмотренные примеры показывают, что при обработке экспериментальных данных нужно очень четко понимать, какой тип импульсных воздействий был реализован при испытаниях. В случае высокоскоростных запороговых импульсов метод определения откольной прочности по „сбросу“ дает аналогичные с волновой методикой результаты. Для пороговых же воздействий значение растягивающего напряжения в момент разрушения может оказаться значительно ниже, а сам момент разрыва может приходиться на ниспадающий участок локальной истории напряжения. В таких ситуациях связывать величину напряжения разрушения со значени- ем напряжения, определенным по методу сброса, уже некорректно. Из-за этой интерпретационной „ошибки“ может получаться значительная разница в результатах, получаемых разными способами.

Отметим также, что при обработке экспериментов по сбросу скорости, как правило, a priori подразумевается существование критического напряжения разрыва, являющегося мерой динамической прочности материала. Величина сброса скорости однозначно связывается с данным напряжением, обычно определяемым как откольная прочность. При таком способе обработки данных испытаний на откол ряд важных эффектов, таких, например, как отмеченный эффект задержанного разрушения, изначально исключается из рассмотрения и теряется. В то же время волновая методика позволяет определить историю напряжений в откольном сечении вплоть до момента разрушения, а значение напряжения в момент разрыва находится по этой методике независимо от выполнения каких-либо предельных условий. При этом стоит отметить, что рассмотренный выше простейший вариант анализа также имеет существенные ограничения к применению, из-за того, что в нем использовалось решение волновой задачи в линейно-упругой постановке. На практике, при динамических испытаниях многих материалов уже на стадии сжатия могут происходить различные необратимые процессы, такие как пластическая деформация или фазовые превращения. Наличие этих явлений приводит к необходимости учета их влияния на уравнения и параметры решаемой задачи (вид волнового уравнения, значения констант, в том числе, скорости распространения волн напряжений в среде, и т. п.). Тем не менее приведенные замечания относительно сопоставления обработки данных испытаний по полному решению волновой задачи с упрощенной методикой по сбросу скорости остаются в силе и в случае более сложных систем, что может существенно повлиять на выводы относительно значений откольной прочности, а также существования тех или иных эффектов динамического разрушения материалов. 


\section{Список литературы}

[1] Н.А. Златин, С.М. Мочалов, Г.С. Пугачев, А.М. Брагов. ФTT 16, 1752 (1974).

[2] K.B. Broberg. Cracks and Fracture. Academic Press, London (1999). $754 \mathrm{c}$.

[3] D.E. Grady, R.E. Hollenbach. Geophys. Res. Lett. 6, 73 (1979).

[4] D.D. Bloomquist, S.A. Sheffield. J. Appl. Phys. 54, 1717 (1983).

[5] L.M. Barker, R.E. Hollenbach. J. Appl. Phys. 43, 4669 (1972).

[6] S. Kubota, Y. Ogata, Y. Wada, G. Simangunsong, H. Shimada, K. Matsui. Int. J. Rock Mech. Mining Sci. 45, 397 (2008).

[7] B. Glam, M. Werdiger, Y. Horovitz, E. Moshe, S.L. Pistinner. Publ. J. Phys. Conf. Ser. 500, 112029 (2014).

[8] A.P. Rybakov. Int. J. Impact Eng. 24, 1041 (2000).

[9] J.P. Cuq-Lelandais, M. Boustie, L. Berthe, T. de Rességuier, P. Combis, J.P. Colombier, M. Nivard, A. Claverie. J. Phys. D 42, 065402 (2009).

[10] E.B. Zaretsky, G.I. Kanel. J. Appl. Phys 112, 053511 (2012).

[11] G.I. Kanel. ФГВ 18, 84 (1982).

[12] Y.V. Petrov, A.A. Utkin. Sov. Mater. Sci. 25, 153 (1989).

[13] Ю.В. Петров, И.В. Смирнов, А.А. Уткин. МТТ 45, 200 (2010).

[14] Н.Ф. Морозов, Ю.В. Петров, А.А. Уткин. ДАН СССР 313, 276 (1990).

[15] Н.А. Златин, Н.Н. Песчанская, Г.С. Пугачев. ЖТФ 56, 403 (1986).

[16] A.N. Berezkin, S.I. Krivosheev, Y.V. Petrov, A.A. Utkin. Doklady Phys. 45, 617 (2000). 